\title{
Evaluation of Wild Dianthus Species as Genetic Resources for Fragrant Carnation Breeding Based on Their Floral Scent Composition
}

\author{
Kyutaro Kishimoto*, Masayoshi Nakayama, Masafumi Yagi, Takashi Onozaki \\ and Naomi Oyama-Okubo
}

National Institute of Floriculture Science, National Agriculture and Food Research Organization (NARO), 2-1 Fujimoto, Tsukuba 305-8519, Japan

\begin{abstract}
Most modern carnation (Dianthus caryophyllus L.) cultivars tend to have little fragrance. Wild Dianthus species having a strong or characteristic fragrance were thought to be promising genomes for the introduction of fragrance into these modern carnation cultivars. We selected 10 plants with fragrant flowers and 1 hardly fragrant plant, which was used as a negative fragrant control, from wild Dianthus gene resources of National Institute of Floriculture Science, and divided them into 4 groups according to their sensory characteristics. Gas chromatography-mass spectrometry (GC-MS) analysis indicated that the scent compounds of wild Dianthus species were principally composed of 18 volatile compounds, which were benzenoids, terpenoids, or fatty acid derivatives. The sweet and medicinal fragrance of Group 1, having the strongest scent, was due to methyl salicylate, a benzenoid. The citrus-like fragrance of Group 2 was due to $\beta$-ocimene and $\beta$-caryophyllene, two terpenoids. The green leafy odor of Group 3 was due to $(Z)$-3-hexenyl acetate, a fatty acid derivative. In Group 4 flower, which had little fragrance, scent compounds were hardly detected. In theses flowers, the composition of the emitted scent compounds differed from that of the endogenous scent compounds; the scent compounds, having a high vapor pressure and a relatively low boiling point, were efficiently emitted from the flower organs. Major benzenoids in D. hungaricus of Group 1 were distributed in petal limbs, and major terpenoids in D. superbus of Group 2 and major fatty acid derivatives in $D$. sp. 2 of Group 3 were distributed in petal bottoms and collectively, in the stamens and pistil. These results demonstrate that the floral tissues which produce fragrance differ for each Dianthus species. In this study, Group 1, being rich in methyl salicylate, and Group 2 , being rich in $\beta$-ocimene and $\beta$-caryophyllene, were suggested important gene resources for introducing fragrance into carnations because these scent compounds have a pleasant smell and contribute strongly to the fragrance features of their respective flowers.
\end{abstract}

Key Words: carnation, Dianthus, floral scent, fragrance, GC-MS analysis.

\section{Introduction}

Over 300 species of Dianthus are distributed in the northern hemisphere in Europe and Asia, including Japan, and other minor species are found in North America and in upland regions in Africa (Galbally and Galbally, 1997). There are over 20 species of wild Dianthus used for breeding carnations (D. caryophyllus L.) and pinks (D. chinensis L. and others) (Galbally and Galbally, 1997). A complex interspecific hybridization program carried out over many years in Europe has led to the breeding of modern carnation cultivars (Segers,

Received; July 9, 2010. Accepted; September 9, 2010.

* Corresponding author (E-mail: cucumber@affrc.go.jp).
1987). It is a remarkable feature of Dianthus plants that interspecific hybridization is easily performed among wild species and horticultural cultivars (Hamilton and Walters, 1989). Wild Dianthus species have been used to introduce novel characters, such as new floral color and shape (Nimura et al., 2006, 2008) or disease resistance (Onozaki et al., 1999), into carnation cultivars.

Floral scent is an important commercial character of ornamental flowers (Dudareva and Pichersky, 2006). Carnations are the most important Dianthus species. Floral scents of several carnation cultivars were reported to be composed of benzenoids, terpenoids, and fatty acid derivatives (Clery et al., 1999; Hudak and Thompson, 1997; Schade et al., 2001; Zuker et al., 2002), among which benzenoids are the most important fragrance 
factors (Clery et al., 1999). Several classical carnation cultivars possess a spicy/clove odor, which is derived from several benzenoids (Clery et al., 1999); oils extracted from these flowers are used as perfume for brilliantines, hair oils, talcum powders, and soaps in Southern Europe and North Africa (Anonis, 1985); however, benzenoid levels are markedly lower in most modern carnation cultivars, resulting in the loss of a strong fragrance (Clery et al., 1999). Wild Dianthus species having a strong or characteristic fragrance are promising materials for introducing fragrance into these modern carnation cultivars.

Ten plants with fragrant flowers and 1 hardly fragrant plant, which was used as a negative fragrant control, were selected from wild Dianthus gene resources of National Institute of Floriculture Science (NIFS) and grouped according to their sensory characteristics. By analyzing the emitted floral scent composition, it was possible to identify key compounds producing the fragrance characterizing each Dianthus group. Each scent compound has a different vapor pressure, and since lower boiling point compounds tend to have a higher vapor pressure, the chemical composition of the emitted fragrance compounds often differs from that of endogenous fragrance compounds in the flower organs (Oyama-Okubo et al., 2005). The endogenous fragrance composition that probably directly reflected the actual state of their biosynthesis activities was investigated. The distribution of the scent compounds was also investigated in each flower organ, and the organs contributing to floral fragrance were revealed. Based on these properties, we discuss the potency of wild Dianthus species as fragrance-breeding materials.

\section{Materials and Methods}

\section{Plant materials}

Dianthus superbus L. and D. superbus floral dwarf mutant (dwarf) were sampled in Hokkaido, Japan (Onozaki, 2001). D. superbus var. superbus f. leucanthus T. Shimizu (96), D. superbus var. speciosus Rchb (95), D. hungaricus Pers (59), D. pyrenaicus Pourret (86), D. banaticus (Heuffel) Borbás (11), D. knappii Ascherson and Kanitz ex Borbás (211), D. sp. 1 (149S2), D. sp. 2 (87), and D. sp. 3 (K119) are maintained as gene resources of NIFS in Tsukuba, Japan. Each number in parentheses is an accession number of NIFS. All of these Dianthus plants were grown in a greenhouse at NIFS under standard growing conditions for the species.

\section{Analysis of emitted floral volatiles}

The volatiles emitted by Dianthus flowers were collected using a dynamic headspace sampling system (Oyama-Okubo et al., 2005). Freshly cut flowering branches were covered with a Tedlar Bag $(500 \mathrm{~mL}$ volume, GL Science, Tokyo, Japan). A constant stream of air filtered through activated charcoal was pumped at a flow rate of $500 \mathrm{~mL} \cdot \mathrm{min}^{-1}$ through the bag. The volatiles were trapped on a Tenax ${ }^{\mathrm{TA}}$ column $(180 \mathrm{mg}$, GL Science) for $1 \mathrm{~h}$ around noon. A thermal desorption system (Gerstel GmbH and Co. KG, Linthicum, USA) was used, in which the desorption temperature was $280^{\circ} \mathrm{C}$ and the time was $1.5 \mathrm{~min}$. Injection was performed in splitless mode, which was used to introduce the trapped volatiles into a gas chromatography-mass spectrometry apparatus (GS-MS) (Agilent 5973, Agilent Technologies, Wilmington, USA).

The GC was equipped with a capillary DB-WAX column $(30 \mathrm{~m} \times 0.25 \mathrm{~mm}$ ID, and $0.25 \mu \mathrm{m}$ film thickness, Agilent Technologies). Helium was used as a carrier gas at a flow rate of $1.0 \mathrm{~mL} \cdot \mathrm{min}^{-1}$. The temperature program of the oven was set to $45^{\circ} \mathrm{C}$ for $2 \mathrm{~min}$, then increased $3^{\circ} \mathrm{C} \cdot \mathrm{min}^{-1}$ up to $220^{\circ} \mathrm{C}$, and kept at this temperature for $10 \mathrm{~min}$. Interface and ion source temperatures were $280^{\circ} \mathrm{C}$ and $250^{\circ} \mathrm{C}$, respectively. Ionization was performed in electron impact mode at $70 \mathrm{eV}$, and a mass scan range of $30-350 \mathrm{~m} / z$ was monitored. Volatile compounds were identified using the NIST02 library search system provided with GC-MS software (Agilent Technologies) and crosschecked by comparing the mass spectrum and retention time with an authentic sample under the same conditions. The amount of each compound was calculated based on the peak area of total ion chromatography.

\section{Analysis of endogenous floral volatiles}

Flowers were collected around noon, and the calyx was removed. Some of the flower samples were separated into petal limbs, petal bottoms, and combined stamens and pistil for analysis of these three separate parts. Whole flowers with the calyx removed were also analyzed. Each sample was crushed in liquid nitrogen and then mixed with $5 \mathrm{~mL} n$-pentane. The mixture was heated in a microwave oven $(700 \mathrm{~W})$ for $20 \mathrm{~s}$. and then ethyl decanoate was added as an internal standard. The extract was dehydrated with anhydrous sodium sulfate and concentrated by heating at $40^{\circ} \mathrm{C}$. The concentrated extract was qualitatively analyzed using GC-MS under the same conditions. Qualitative analysis of each volatile compound was performed using a GC system (Agilent 6890, Agilent Technologies) under the same conditions as GC-MS, and was monitored with a flame ionization detector. The amount of each volatile compound was calculated by comparison with the peak area of the internal standard.

\section{Results}

\section{Grouping of Dianthus plants based on their sensory} fragrance

Wild Dianthus flowers were divided into 4 groups according to the sensory characteristics of their fragrance (Fig. 1). Group 1, which contained D.hungaricus, D. pyrenaicus, and $D$. sp. 1, had the strongest fragrance, characterized as a medicinal fragrance. Group 2 , which has a citrus-like fragrance, was composed of 


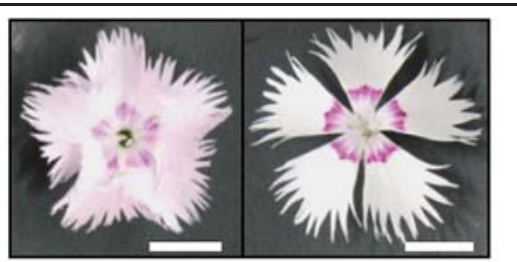

Group 1

D. hungaricus (59) D. pyrenaicus (86)
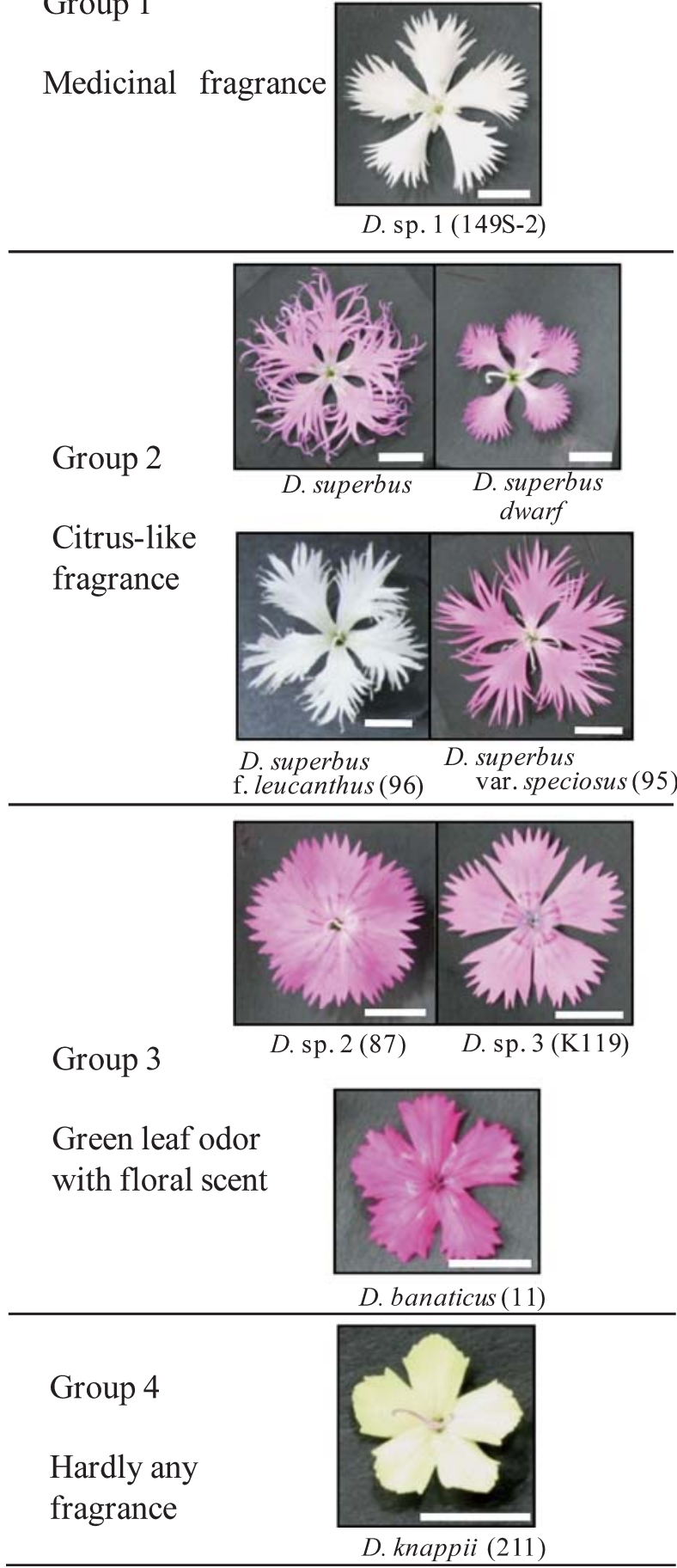

Fig. 1. Dianthus flowers analyzed in this study. Each number in parentheses is an accession number of the National Institute of Floriculture Science. Bar $=10 \mathrm{~mm}$. four $\quad$ D. superbus lines: D. superbus, D. superbus f.leucanthus, D. superbus var. speciosus, and D. superbus floral dwarf mutant (dwarf). Group 3, with a green leafy odor with floral scent, contained $D$. sp. 2, $D$. sp. 3, and D. banaticus. Group 4 which hardly had any scent, contained D. knappii.

\section{Composition of the emitted floral scent}

The floral volatiles were trapped using the dynamic head space method with a Tenax ${ }^{\mathrm{TA}}$ column and then analyzed using GC-MS. The floral scents were composed of benzenoids, terpenoids, and fatty acid derivatives, and 18 fragrance compounds were identified as typical components (Table 1 ).

In Group 1, the dominant emitted scent compounds were benzenoids, among which methyl salicylate and isoeugenol were common major components of all three species. In Group 2, the dominant emitted scent compounds were terpenoids, among which $\beta$-ocimene, a monoterpene, and $\beta$-caryophyllene, a sesquiterpene, were major components (Table 1). D. superbus var. speciosus is an alpine variety, and its flower color was slightly deeper than that of D. superbus (Fig. 1). D. superbus f.leucanthus is a white flower forma and D. superbus dwarf is a dwarfed flower mutant (Fig. 1). Although flower color or shape differed in these of $D$. superbus lines, a significant difference could not be found in the composition and amount of floral scents. In Group 3, the common major chemicals were fatty acid derivatives, among which ( $Z$ )-3-hexenyl acetate was a principal component. In Group 4, small amounts of scent compounds were detected (Table 1).

\section{Composition of the endogenous floral scent}

The endogenous scent compounds of Dianthus flowers extracted with $n$-pentane were analyzed using a GC. In Group 1, the dominant endogenous scent compounds were benzenoids (Table 2), similar to the emitted scent compounds. Isoeugenol, which is a common major emitted scent compound, was also a common major component in the endogenous scent compounds. Methyl salicylate, another common major emitted scent compound, was not detected as a major endogenous scent compound in any Group 1 plants. On the other hand, benzyl alcohol, benzyl benzoate, or cinnamyl alcohol, which were minor emitted scent compounds, were often detected as major endogenous scent compounds (Table 2). In Group 2, the dominant endogenous scent compounds were terpenoids (Table 2), similar to the emitted scent compounds. $\beta$ Caryophyllene, which was a common major emitted scent compound, was also a major component of the endogenous scent compounds, and $\alpha$-caryophyllene followed suit in Group 2 plants. The amount of $\beta$ ocimene, which was another common major emitted scent compound, was about $2 \%$ of that of $\beta$ caryophyllene (Table 2). In Group 3, the dominant 


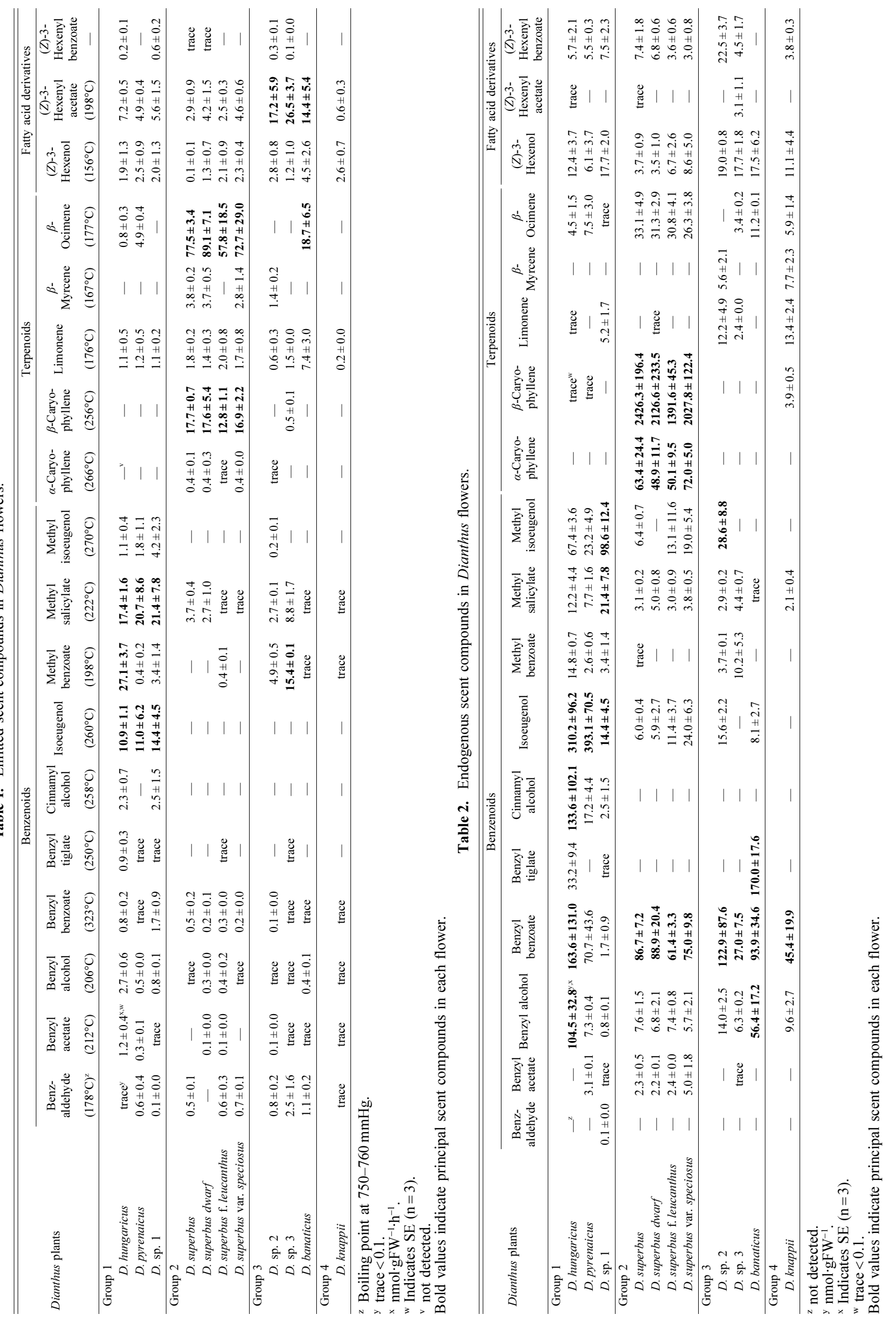


chemicals were benzenoids such as benzyl benzoate (Table 2). (Z)-3-Hexenyl acetate, which was the major emitted scent compound, was not, or was only slightly detected, its precursor $(Z)$-3-hexenol was detected in the range of $17-20 \mathrm{nmol}^{\circ} \mathrm{gFW}^{-1}$ (Table 2). In Group 4, benzyl benzoate was the principal endogenous compound (Table 2).

Interestingly, although benzyl benzoate accumulated in tissue at high levels in plants of all groups (Table 2), only small amounts were emitted, whereas benzaldehyde was detected in the emitted scent compounds from almost all plants, but was not detected in the endogenous compounds of any plant (Table 2).

\section{Distribution of the floral scent}

In order to clarify the distribution of the scent compounds in the flower organs of Dianthus, the endogenous scent amounts in petal limbs, petal bottoms and collectively, the stamens and pistil of D. hungaricus, D. superbus, and D. sp. 2 (Fig. 2), which were representative plants in each of the Groups 1-3, were compared. In D. hungaricus (Group 1), methyl salicylate, cinnamyl alcohol, and isoeugenol, which were the major emitted and endogenous scent compounds (Tables 1 and 2 ), were distributed in the petal limbs (Table 3 ). In contrast, the amounts of benzyl tiglate and (Z)-3-hexenol were lower in the petal limbs than in the petal bottoms and in the combined stamens and pistil (Table 3 ). In D. superbus (Group 2), $\beta$-caryophyllene, which was a
Combined stamens and pistil

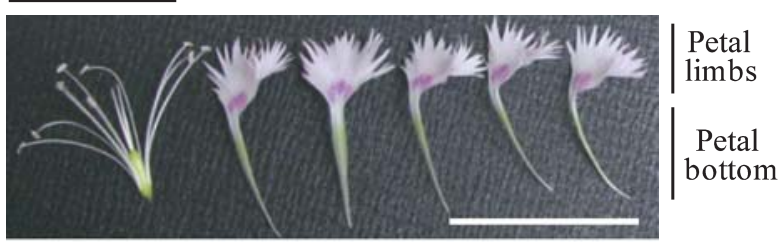

\section{D. hungaricus (Group 1)}

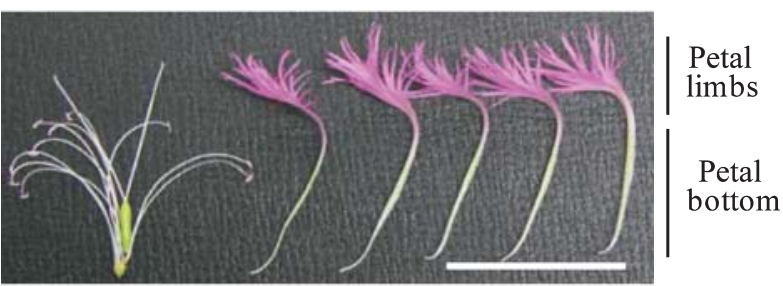

D. superbus (Group 2)

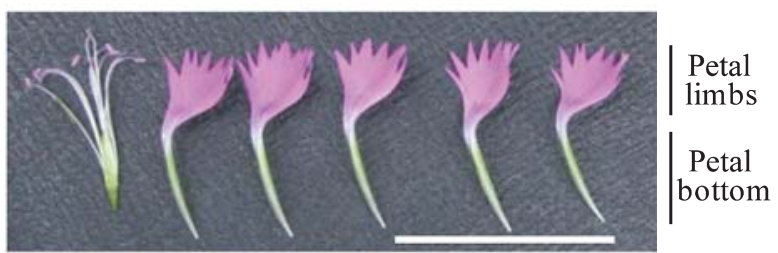

D. sp. 2 (Group 3)

Fig. 2. Organs investigated in Dianthus flowers. Flowers were separated into combined stamens and pistil, petal limbs, and petal bottoms. Bars $=3 \mathrm{~cm}$.

Table 3. Distribution of scent compounds in Dianthus flowers.

\begin{tabular}{|c|c|c|c|c|c|c|c|c|c|c|}
\hline \multirow[b]{2}{*}{ Compounds } & & \multicolumn{3}{|c|}{ D. hungaricus (Group1) } & \multicolumn{3}{|c|}{ D. superbus (Group 2) } & \multicolumn{3}{|c|}{ D. sp. 2 (Group 3) } \\
\hline & & Petal limb & Petal tube & $\begin{array}{l}\text { Combined } \\
\text { stamens } \\
\text { and pistil }\end{array}$ & Petal limb & Petal tube & $\begin{array}{l}\text { Combined } \\
\text { stamens } \\
\text { and pistil }\end{array}$ & Petal limb & Petal tube & $\begin{array}{l}\text { Combined } \\
\text { stamens } \\
\text { and pistil }\end{array}$ \\
\hline \multirow{10}{*}{ Benzenoids } & Benzaldehyde & trace $^{2}$ & trace & $-y$ & - & - & - & - & - & - \\
\hline & Benzyl acetate & - & trace & - & - & $9.6 \pm 3.8^{\mathrm{x}, \mathrm{w}}$ & $3.3 \pm 0.5$ & - & - & - \\
\hline & Benzyl alcohol & $136.6 \pm 9.5$ & $21.7 \pm 1.2$ & $23.1 \pm 1.6$ & $15.4 \pm 2.9$ & $15.9 \pm 3.6$ & $15.0 \pm 2.6$ & $4.4 \pm 0.7$ & $15.0 \pm 1.6$ & $9.2 \pm 2.4$ \\
\hline & Benzyl benzoate & $127.4 \pm 27.8$ & $131.3 \pm 20.5$ & $147.4 \pm 31.9$ & $44.6 \pm 22.5$ & $80.9 \pm 18.7$ & $61.2 \pm 16.9$ & $87.8 \pm 27.2$ & $80.0 \pm 21.6$ & $114.1 \pm 57.2$ \\
\hline & Benzyl tiglate & - & $71.2 \pm 24.1$ & $20.5 \pm 5.1$ & - & - & - & - & - & - \\
\hline & Cinnamyl alcohol & $149.9 \pm 21.8$ & $4.0 \pm 0.5$ & trace & - & - & - & - & $3.3 \pm 1.2$ & - \\
\hline & Isoeugenol & $691.4 \pm 179.2$ & $6.4 \pm 3.0$ & $23.2 \pm 7.8$ & $13.9 \pm 3.3$ & - & - & $2.4 \pm 0.6$ & $14.4 \pm 5.6$ & - \\
\hline & Methyl benzoate & $15.6 \pm 6.6$ & $10.9 \pm 1.2$ & - & $8.4 \pm 3.6$ & - & - & $2.2 \pm 0.3$ & $10.1 \pm 0.5$ & - \\
\hline & Methyl salicylate & $16.0 \pm 0.9$ & trace & - & $5.9 \pm 0.5$ & trace & trace & $3.8 \pm 0.9$ & - & - \\
\hline & Methyl isoeugenol & $64.4 \pm 16.8$ & $33.5 \pm 0.8$ & $37.3 \pm 11.0$ & $9.5 \pm 2.2$ & $8.9 \pm 4.0$ & $4.7 \pm 0.8$ & $13.8 \pm 1.0$ & $28.1 \pm 8.8$ & - \\
\hline \multirow[t]{5}{*}{ Terpenoids } & $\alpha$-Caryophyllene & - & - & - & - & $99.9 \pm 20.0$ & $28.9 \pm 4.8$ & - & - & - \\
\hline & $\beta$-Caryophyllene & trace & trace & - & $48.3 \pm 28.5$ & $3149.6 \pm 738.9$ & $709.3 \pm 75.2$ & - & - & - \\
\hline & Limonene & trace & - & - & - & - & - & trace & - & $16.6 \pm 2.3$ \\
\hline & $\beta$-Myrcene & - & - & - & - & - & - & - & - & $6.0 \pm 0.2$ \\
\hline & $\beta$-Ocimene & $11.0 \pm 4.8$ & trace & - & $22.7 \pm 4.8$ & $42.0 \pm 6.8$ & $26.1 \pm 8.2$ & trace & - & - \\
\hline \multirow{3}{*}{$\begin{array}{l}\text { Fatty acid } \\
\text { derivatives }\end{array}$} & (Z)-3-Hexenol & $5.4 \pm 1.1$ & $18.1 \pm 12.1$ & $19.3 \pm 11.2$ & - & $5.4 \pm 3.4$ & $5.0 \pm 1.8$ & $5.8 \pm 0.6$ & $21.4 \pm 6.4$ & $28.2 \pm 1.4$ \\
\hline & (Z)-3-Hexenyl acetate & trace & - & - & - & - & - & - & - & trace \\
\hline & (Z)-3-Hexenyl benzoate & - & $8.5 \pm 3.3$ & - & - & - & $13.8 \pm 0.4$ & $10.8 \pm 1.8$ & $10.6 \pm 1.6$ & $5.3 \pm 0.3$ \\
\hline
\end{tabular}

\footnotetext{
${ }^{z}$ trace $<0.1$.

${ }^{y}$ not detected

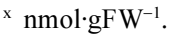

${ }^{\mathrm{w}}$ Indicates SE $(\mathrm{n}=3)$.

Bold values indicate principal scent compounds in each flower.
} 
major component of both emitted and endogenous scent compounds, was mostly distributed in the petal bottoms and in the combined stamens and pistil, which was also the case for $\alpha$-caryophyllene, which was another major endogenous scent compound. The amount of $\beta$-ocimene, which was another major emitted scent compound, was about 2 times higher in the petal bottoms than in the petal limbs and the combined stamens and pistil (Table 3). On the other hand, the amounts of several minor benzenoids; isoeugenol, methyl salicylate, and methyl benzoate, were comparatively higher in petal limbs than in the other organs (Table 3 ). In $D$. sp. 2 (Group 3), the amount of (Z)-3-hexenol, which was a precursor of (Z)-3-hexenyl acetate, was higher in petal bottoms and in the combined stamens and pistil than in petal limbs (Table 3). The amounts of benzyl alcohol, isoeugenol, methyl benzoate, and methyl isoeugenol were 2-4 times higher in petal bottoms than in petal limbs (Table 3). Benzyl benzoate was detected in all flower organs and did not show any specific distribution (Table 3).

\section{Discussion}

Floral scents are produced by complexes of various chemical compounds, and benzenoids/phenylpropanoids, terpenoids, fatty acid derivatives, carotenoid derivatives, and nitrogen- or sulfur-containing compounds are known to be floral scent components (Dudareva and Pichersky, 2006). This study indicates that the scents of Dianthus wild species were composed of three components; benzenoids, terpenoids, and fatty acid derivatives.

Group 1 was the most fragrant group and is expected to be the most promising fragrance breeding material. The strong fragrance of their flowers is due to emissions rich in benzenoids, where benzenoids were also the almost exclusive endogenous scent component, suggesting the active biosynthesis of benzenoids rather than other scent components. In this group, methyl salicylate and isoeugenol were common major scent compounds, as methyl benzoate also was in D. hungaricus. The threshold concentration of methyl salicylate for aroma sensing is $40 \mathrm{ppb}$, which is about 2.5-2.8 times lower than that required for the detection of isoeugenol and methyl benzoate (Burdock, 2010). Thus, we conclude that methyl salicylate is the predominant contributor to the fragrance of Group 1, and isoeugenol and methylbenzoate are secondary contributors. Methyl salicylate is generally used as a fragrance material in foods, beverages, and pharmaceuticals, and its scent is often perceived to be a sweet or medicinal (Burdock, 2010; Clery et al., 1999). As far as we know, carnation cultivars having rich methyl salicylate have not been reported in academic research. The scent of isoeugenol is a representative floral fragrance of carnations, and is perceived as being spicy (Burdock, 2010). Methyl benzoate, which is one of the major carnation fragrance compounds (Zuker et al., 2002), is perceived as being phenolic (Burdock, 2010). Thus, the fragrance features of Group 1 are strong sweet and medicinal, which is different from that of carnations, so that the plants in Group 1 are assessed to be breeding stock with the potential to enhance the fragrance of carnations by adding the new methyl salicylate fragrance.

In Group 2, two terpenoids, $\beta$-caryophyllene and $\beta$ ocimene, were major scent compounds that are also found in carnation scents as minor components (Clery et al., 1999). $\beta$-Caryophyllene is a bicyclic sesquiterpene and is included in the essential oils of many flowers, and its relaxing odor is often described as being woodyspicy, dry or camphoraceous, with a citrus background (Burdock, 2010). $\beta$-Ocimene is found in various flowers and fruits, and is described as being a warm herbaceous odor, or woody with a floral scent (Burdock, 2010). The fragrance is due to these two terpenoids being emitted from their flowers, where terpenoids also occupied a large part of the endogenous scent component, suggesting that the biosynthesis of terpenoids occurs actively, rather than that of other scent components. So far in Dianthus plants, terpenoids have only been found as the dominant scent compounds in D. superbus plants, and not in other Dianthus species or carnations (Clery et al., 1999; Hudak et al., 1997; Schade et al., 2001; Zuker et al., 2002). Thus, D. superbus is considered to be a breeding material with the potential to add a novel terpenoid fragrance to carnations.

In Group 3, a fatty acid derivative, (Z)-3-hexenyl acetate, was detected as a common major emitted scent compound. Thus, this compound is considered to be the most important fragrance component. The scent of $(Z)$ 3-hexenyl acetate is described as being green, fruity, and with a floral note reminiscent of banana (Burdock, 2010). In some carnation flowers, C6-aldehydes and C6alcohols, which are precursors of (Z)-3-hexenyl acetate, were reported as the major volatiles (Schade et al., 2001). The distinctive green odor of crushed leaves is due to these C6-volatiles and their esters (Matsui, 2006). The green odor derived from ( $Z$ )-3-hexenyl acetate appears to be the major fragrance feature of Group 3. Group 4, $D$. knappii, hardly had sensory fragrance. Predictably, an emitted fragrance compound was hardly detected in this flower.

In Group 1, although methyl salicylate and isoeugenol are the major emitted scent compounds, the endogenous amount of methyl salicylate was markedly less than that of isoeugenol. This result indicates that methyl salicylate has a higher vapor pressure, and is emitted more efficiently than isoeugenol. A similar phenomenon is found with $\beta$-ocimene and (Z)-3-hexehnyl acetate in Groups 2 and 3, respectively, in that these compounds have higher vapor pressures than the other detected major scent compounds. We found that the emission efficiencies of volatiles from flower organs are negatively related to the boiling point of the volatiles in 
Petunia flowers (Oyama-Okubo et al., 2005). This result in Dianthus flowers coincided with Petunia flowers, methyl salicylate, $\beta$-ocimene, and (Z)-3-hexehnyl acetate each have a lower boiling point than the other major scent compounds found, and conversely, it was found that benzyl alcohol, benzyl benzoate, and cinnamyl alcohol each had a higher boiling point and lower vapor pressure. These higher vapor pressure compounds are also thought to efficiently contribute to the floral fragrance of Dianthus plants.

In a carnation cultivar, 'Eilat', the floral scent was suggested to negatively cross talk with floral color (Zuker et al., 2002); however, the difference in floral color among D. superbus plants had little effect on the floral scent. In addition, the morphological differences in petal limbs had little effect on the floral scent. On the other hand, distribution analysis of fragrance compounds in flower organs indicated a different relationship between the morphology of flower and fragrance. In D. hungaricus, the major benzenoids, including methyl salicylate and isoeugenol, were distributed in petal limbs, suggesting that the fragrance may be enhanced by double-flowering. In D. superbus and D. sp. 2, the major fragrance compounds were distributed in petal bottoms and in the combined stamens and pistil. These findings indicate that these inconspicuous organs also should be considered when breeding for fragrance.

In this study, we determined that D.hungaricus, D. pyrenaicus, and D. sp. 1 of Group 1 and D. superbus of Group 2 are promising wild Dianthus plants as gene resources to add new fragrance to carnations. Increasing the amounts of methyl salicylate and terpenoids biosynthesized in these plants would probably be a valuable target for fragrance breeding. We plan to further evaluate these wild Dianthus species for fragrant carnation breeding by studying the manner of inheritance of the emitted and endogenous scent compounds in interspecific hybridization with carnation.

\section{Literature Cited}

Anonis, D. P. 1985. The application of carnation in perfumery. Flav. Fragr. J. 1: 9-15.

Burdock, G. H. 2010. Fenaroli's handbook of flavor ingredients six edition. CRC Press, Boca Raton.

Clery, R. A., N. E. Owen and S. F. Chambers. 1999. An investigation into the scent of carnations. J. Essent. Oil Res. 11: 355-359.

Dudareva, N. and E. Pichersky. 2006. Biology of floral scent. CRC Press, Boca Raton.

Galbally, J. and E. Galbally. 1997. Carnation and pinks for garden and greenhouse. Timber Press, Portland.

Hamilton, R. F. L. and S. M. Walters. 1989. Dianthus Linnaeus. p. 185-191. In: S. M. Walters, J. C. M. Alexander, A. Brady, C. D. Brickell, J. Cullen, P. S. Green, V. H. Heywood, V. A. Matthews, N. K. B. Robson, P. F. Yeo and S. G. Knees (eds.). The European garden flora Vol. 3. Cambridge University Press, Cambridge.

Hudak, K. H. and J. E. Thompson. 1997. Subcellular localization of secondary lipid metabolites including fragrance volatiles in carnation petals. Plant Physiol. 114: 705-713.

Matsui, K. 2006. Green leaf volatiles: hydroperoxide lyase pathway of oxylipin metabolism. Curr. Opin. Plant Biol. 9: 274-280.

Nimura, M., J. Kato and M. Mii. 2006. Interspecific hybrid production by reciprocal crosses between Dianthus caryophyllus L. and Dianthus $\times$ isensis Hirahata et Kitamura. J. Hort. Sci. Biotech. 81: 995-1001.

Nimura, M., J. Kato, M. Mii and K. Ohishi. 2008. Crosscompatibility and the polyploidy of progenies in reciprocal backcrosses between diploid carnation (Dianthus caryophyllus L.) and its amphidiploid with Dianthus japonicus Thunb. Sci. Hortic. 115: 183-189.

Onozaki, T. 2001. Exploration and collection of Dianthus superbus var. longicalicinus and D. superbus in Mie prefecture and Hokkaido. Annual Report on Exploration and Introduction of Plant Genetic Resources (Japan) 17: 49-54 (In Japanese with English abstract).

Onozaki, T., T. Yamaguchi, M. Himeno and H. Ikeda. 1999. Evaluation of wild Dianthus accessions for resistance to bacterial wilt (Pseudomonas caryophylli). J. Japan. Soc. Hort. Sci. 68: 974-978.

Oyama-Okubo, N., T. Ando, N. Watanabe, E. Marchesi, K. Uchida and N. Nakayama. 2005. Emission mechanism of floral scent in Petunia axillaries. Biosci. Biotechnol. Biochem. 69: 773777.

Schade, F., R. L. Legge and J. E. Thompson. 2001. Fragrance volatiles of developing and senescing carnation flowers. Phytochemistry 56: 703-710.

Segers, A. 1987. The development of interspecific carnation hybrids. Acta Hort. 216: 373-375.

Zuker, A., T. Tzfira, H. Ben-Meir, M. Ovadis, E. Shklarman, H. Itzhaki, G. Forkmann, S. Martens, I. Neta-Sharir, D. Weiss and A. Vainstein. 2002. Modification of flower color and fragrance by antisense suppression of the flavanone 3hydroxylase gene. Mol. Breeding. 9: 33-41. 